\title{
Formación tecnológica y universidad pública en Colombia ${ }^{1}$
}

\author{
Technology training and public university in Colombia \\ Training tecnologia e universidade pública na Colômbia
}

Recibido: septiembre de 2012

Aceptado: diciembre de 2012

Mirna Jirón Popova ${ }^{2}$

\section{Resumen}

En Colombia las preocupaciones por modernizar la Educación Superior emergen a mediados de la segunda mitad del siglo XX, como respuesta a las recomendaciones de los organismos internacionales de asumir el Desarrollo Económico como meta. La modernización adoptó diversas estrategias. Una de estas fue orientar la Educación Superior hacia modalidades cortas de formación. En ese sentido, este artículo plantea una mirada a los acontecimientos que tuvieron lugar en los ámbitos regional y nacional y que propiciaron la institucionalización de la Formación Tecnológica. Además, se exponen las razones por las cuales algunas universidades públicas del país se adhirieron a la oferta de esos programas entre los años sesenta y ochenta.

Palabras clave: modernización, educación superior, formación tecnológica, segunda mitad del siglo XX, Colombia.

\begin{abstract}
In Colombia the concerns for the update of higher education emerge on the second half of the 20th century, as a response at the suggestions of international organisms of assuming Economic development as a goal. The updating adopted several strategies. One of them was to aim higher education at short formation modalities. In that sense, this article considers a view at the events that took place in the regional and national settings, which fostered the institutionalization of the Technological formation. Besides, we present the reasons why some public universities in the country agreed with the offer of those programs in the decades among years 60 and 80 .
\end{abstract}

Keywords: modernization, higher education, technology education, second half of 20th, Colombia, century.

1 Artículo de investigación derivado de la Tesis doctoral realizada en el Doctorado Interinstitucional en Educación de la Universidad Distrital Francisco José de Caldas.

2 Docente Facultad Tecnológica, Universidad Distrital Francisco José de Caldas, Bogotá - Colombia. Contacto: mjiron@udistrital.edu.co 


\section{Resumo}

$\mathrm{Na}$ Colômbia as preocupações por modernizar a educação superior emergem em meados da segunda metade do século XX como resposta às recomendações dos organismos internacionais de se assumir o desenvolvimento econômico como meta. A modernização assumiu diversas estratégias. Uma delas foi orientar a educação superior para modalidades de curta formação. Nesse sentido, neste articulo, se oferece um olhar sobre os acontecimentos regionais e nacionais que propiciaram a institucionalização da formação tecnológica e sobre as razões pelas quais algumas universidades públicas do país aderiram, nas décadas dos anos 60 y 80 , à oferta desses programas.

Palavras-chave: modernização, educação superior, formação tecnológica, segunda metade do século XX, Colômbia.

\section{Introducción}

Los estudios sobre la educación superior en Colombia y, específicamente, los relacionados con los procesos de modernización que se suceden en el ámbito regional y nacional son el objeto de la línea de investigación educación superior y Representaciones Sociales -línea de carácter histórico- cuyos propósitos se encaminan a “(...) hacer un acercamiento a los documentos y archivos existentes que permitan estudiar la educación superior en las temáticas específicas abordadas y establecer una nueva mirada desde los acontecimientos y rupturas (...) [para] disponer de un acervo de conocimientos de este fenómeno". Situamos los estudios entre 1950 y 2008, periodo en el cual un nuevo tipo de organización jurídica se da en el contexto internacional y nacional. En América Latina los Estados nacionales se involucran en una nueva "fase de desarrollo", en medio de las tensiones socioeconómicas del momento, situación que se expresa con gran fuerza en los años sesenta.

La universidad por ser una institución de carácter urbano será el espacio de influencia y resonancia de estos cambios, que también parecen generar malestar y producir rupturas. La nueva industria requiere ingenieros de diferentes ramas, obreros especializados, el sector privado requiere profesionales en las áreas de economía, administración, contaduría, etc.; surge así la expansión y la diversificación de carreras en la universidad, el aumento de instituciones de educación superior en las regiones con desarrollos desiguales y el ingreso de nuevos grupos sociales que hacen presión por educación universitaria. Con el Decreto 80 de 1980 y la Ley 30 de 1992 el sistema de educación superior se reorganiza y los estudios técnicos y tecnológicos se configuran como niveles de la educación superior (Ortiz, s.f., pp. 1-3), cuyos “(...) diseños [acortaron] la duración de las carreras profesionales [para] formar personal técnico de nivel superior requerido por la economía del país" (Diario Oficial 34712,1976$)$ y, en especial, por los procesos de modernización y expansión industrial.

Es entonces sobre el nivel tecnológico (formación tecnológica) en Colombia que vuelve la mirada el proyecto de investigación "La formación tecnológica en Colombia en el ámbito de la universidad pública" y, específicamente, sobre las orientaciones de los organismos internacionales en materia de educación superior, la institucionalización de la formación tecnológica y las motivaciones de las universidades públicas por incursionar en la oferta de estos programas ${ }^{3}$.

3 Además, el estudio se propuso extenderla mirada hasta el año 2011, para observar en la universidad pública la coyuntura actual de la formación tecnológica - particularmente en áreas de las tecnologías asociadas a las ingenierías-, en relación con los conceptos, los perfiles y los planes de estudio que se privilegian, y para evidenciar las coyunturas que se desenvuelven en las instituciones -en términos de adaptación y resistencias-, a propósito del despliegue de los mecanismos de control por parte del Estado en cada momento histórico y, en especial, en la vigencia de las normas que reglamentan la Formación por Ciclos Propedéuticos (FpCP) (Ley 749 de 2002) y las disposiciones en materia de condiciones mínimas de calidad de los programas (Decreto 2566 de 2003, Ley 1188 de 2008 y Decreto 1295 de 2010). 


\section{Metodología}

La investigación asumió una perspectiva cualitativa de corte histórico centrada en la observación documental -que es “(...) aquella que se realiza sobre los documentos en los cuales los fenómenos sociales dejan huella (...)" (Duverger, 1962, p. 115)-, para observar los acontecimientos que se sucedieron en el país en relación con la formación tecnológica, pues los documentos son “(...) contenedores de fenómenos sociales que dejan huella (García, s.f.), y un protocolo de investigación que se desplegó en cuatro etapas. En la etapa documental se realizó la búsqueda de fuentes, su sistematización y obtención (impresa o digital), y el trabajo de campo -que se llevó a cabo en un conjunto de universidades públicas ${ }^{4}$ que ofrecen formación tecnológica (FT) en el país desde finales de los años sesenta- tuvo como finalidad ampliar el archivo documental con los testimonios de los actores sociales de la formación tecnológica (coordinadores de programa o directores de escuela, profesores que participaron de la creación o conceptualización de los programas, etc.) mediante la técnica de entrevista semiestructurada e información institucional.

En la etapa textual se pasó al análisis de contenido -entendido como una forma particular de la semántica cuantitativa que atiende a las ideas expresadas y, en consecuencia, “(...) estudia los significados que se expresan en frases enteras: 'El análisis de contenido se caracteriza por el hecho de que las unidades analizadas no son generalmente las palabras, sino los significados (...)' agrupados en cierto número de categorías preestablecidas (...)" (García, s.f.) - con ayuda del software AtlasTi; como resultado de este análisis se obtuvieron 6.932 citas, 1.044 códigos y se crearon 31 familias de documentos y 33 familias de códigos (categorías) que fundamentaron la etapa conceptual, en la cual se llevaron a cabo las actividades de asociaciones y análisis para abocarse -la última etapa de elaboración de productos- a la reconstrucción de la historia de la formación tecnológica y, en especial, para develar sus orígenes en las universidades públicas del país.

\section{Una modalidad de educación superior para el desarrollo}

Con la finalización de la Segunda Guerra Mundial y el establecimiento de un orden geopolítico tripolar - potencias del primer mundo (naciones industrializadas libres) y el segundo mundo (naciones comunistas industrializadas) y países del tercer mundo (naciones pobres no industrializadas)-, los propósitos expansionistas de los Estado Unidos en Latinoamérica y de contención al avance del comunismo en la región no dieron espera. Con ese telón de fondo, se despliegan una serie estudios diagnósticos que pusieron al descubierto un sinnúmero de problemas; uno de ellos -aquel que los expertos de los organismos internacionales consideraron de mayor repercusión en el subdesarrollo- fue la "pobreza”. Así, los expertos iban de país en país elaborando sus teorías sobre la "pobreza" y emitiendo juicios sobre “(...) los 'problemas' (...) creando numerosas categorías de 'cliente'. El desarrollo avanzó creando 'anormalidades' (como 'iletrados', 'subdesarrollados', 'malnutridos', 'pequeños agricultores' o 'campesinos sin tierra')” (Escobar, 1998, p. 89).

Colombia fue el primer país de Latinoamérica que recibió la

(...) famosa en la historia del desarrollo [del país], la Misión Currie. Su nombre mismo ya permite situar al desarrollo dentro de la larga estela que la "misión civilizadora" europea ha dejado en nuestro continente. Esta Misión, patrocinada por el Banco Internacional de Reconstrucción y Fomento, visitó a Colombia entre el 11 de julio y el 5 de noviembre de 1949, con el propósito de formular un programa comprehensivo de desarrollo para nuestro país. La Misión incluía catorce expertos internacionales en las siguientes áreas: agricultura, salud, economía, finanzas públicas y banca, balanza de pagos, industria y energía, petróleo, trasporte y servicios comunales (...) Nunca antes había sido Colombia objeto de

4 Universidades Tecnológica de Pereira, Distrital Francisco José de Caldas, Pedagógica y Tecnológica de Colombia, del Valle, Industrial de Santander, Francisco de Paula Santander. 
un programa tan comprehensivo e integrado; nunca antes se había experimentado en el país con las formas de planificación y organización que el programa sugería. Nunca antes había sido la economía y la sociedad colombianas sujetas a tan detallado escrutinio, a un sondeo tan racional de la vida del país (Escobar, 1996, pp. 13-14).

\section{Entonces, si el}

(...) rasgo esencial del Tercer Mundo era su Pobreza (...) la solución radicaba en el crecimiento económico, lo que implicaba necesariamente el desarrollo como meta (...). Este proceso de "desarrollización" del Tercer Mundo llevará a una "progresiva construcción de un régimen de discurso y práctica en el cual ciertas medidas para la erradicación de la pobreza se volvieron indispensables para el orden mundial" (Martínez, 2004, p. 59). Desarrollarse supondría administrar la "Pobreza" y, con ello, la "(...) intervención en educación, salud, higiene, moralidad, empleo, la enseñanza de buenos hábitos de asociación, ahorro, crianza de los hijos, y así sucesivamente (...)" (Escobar, 1998, p. 54).

También implicaría seguir la lógica del desarrollo de los países del primer mundo-y del programa de ayudas de la Alianza para el Progreso, en el marco de la cual Colombia jugó el papel de "vitrina" (Centro de Estudios de Estadounidenses et al., 2011, p. 11) que debería mostrar los beneficios de las constantes inyecciones de capital extranjero en la aceleración de los procesos de industrialización, la modernización de la industria nacional y la diversificación del aparato productivo nacional con base en la trasferencia de tecnologías, en el marco de la política científica-tecnológica norteamericana (Programa de Paz y Libertad, 1949) en beneficio de los países del Tercer Mundo.

Así, la industrialización no se fundamentó en “(...) una política de elevación de los niveles de desarrollo científico hasta el más alto grado -para hacer posible su asimilación y difusión culturales, al nivel de las sociedades latinoamericanas-, sino que es una consolidación de la dependencia por medio de las modernas estructuras industriales que operan los consorcios y conglomerados norteamericanos en la América Latina" (García, 2006, p. 345) y la tecnología moderna surge

(...) en Colombia y en América Latina, no como resultado de un proceso endógeno de producción autóctona de aquella, ligada a un desarrollo también autóctono de formas de producción (como fue el caso de Europa y en Estados Unidos), sino como un componente implícitamente contenido en los inventos extranjeros que se iban incorporando a la vida de nuestros países en su difícil y lento desarrollo para integrarse a la economía mundial y para ampliar su actividad económica. La enseñanza y el ejercicio de la ingeniería surgieron como un requisito interno para poder aplicar la tecnología que se importaba (Poveda, 1993, p. 18).

Entonces había que fijar para la educación otros propósitos, unos que garantizaran el aumento de la productividad del grueso de la población. Es por ello que la educación en general, pero en particular la educación superior (ES), pasa a engrosar la lista de temas prioritarios de los tecnócratas encargados de delinear las políticas en esa materia, pues era claro que el país requería de"(...) empresarios, ingenieros y técnicos de alta formación, [pero sobre todo de] obreros eficientes y mandos medios para todos los procesos administrativos y fabriles, que no podían suministrar las tradicionales escuelas de artes y oficios que funcionaban en algunas ciudades del país" (Jaramillo, 1989, p. 101); en síntesis, además de modernizar la industria, había que modernizar la educación, para garantizar su "masificación"; pero una masificación que no fuera “(...) en contrasentido de la estratificación social (...) que se refuerza a través de (...) la multiplicación de instituciones de diversa calidad y modalidad educativa, lo que permite que cada estrato social adquiera el capital cultural deseado y se ubique 
posteriormente en el segmento de mercado correspondiente" (Orozco, 1994, p. 34).

\section{Modernización de la educación superior}

A finales de la década de los sesenta, articulado a la lógica del desarrollo - de desplegar la política de conocimiento para diagnosticar problemas y formular políticas (Escobar, 1998, p. 97)-, el gobierno de la época recurre a la Misión de la Universidad de California (1966-1967) y a otra, encabezada por Rudolph P. Atcon, para llevar a cabo un estudio diagnóstico sobre la situación de la ES en el país. Estas misiones inauguran el embate modernizador, dan impulso a las carreras cortas y determinan su adhesión a las universidades pública, pues la “(...) educación superior constituye la verdadera encrucijada en el desarrollo de América Latina (...) la universidad es la legítima formadora del pensamiento de la comunidad (...) sus actividades deben dirigirse hacia (...) b) La preparación de profesionales en número adecuado para las necesidades correspondientes de la sociedad; c) el entrenamiento especializado en técnicas y tecnologías para el desarrollo industrial de la sociedad (...)" (Banco Mundial, 2009).

Así, una “(...) nueva fase de la universidad modernizante nació con los programas de la Alianza para el Progreso. La universidad tradicional no llenaba las ambiciones del país dominante (Estados Unidos) al cual, en la nueva repartición del mundo, le había correspondido (...)” (Molina, 2008, p. 135). “(...) América Latina [que] necesita en grandes cantidades hombres especializados, hombres con iniciativa, con imaginación y con conocimientos técnicos dentro de los más diversos campos del conocimiento y del esfuerzo humano; y los necesita más urgentemente que las máquinas (...)"(Banco Mundial, 2009). Entonces, la universidad debería adoptar una serie de medidas “(...) fundamentales para lograr un avance significativo en la prestación de este servicio, avance que debe traducirse en una mejor educación universitaria para un mayor número de colombianos" (Asociación Colombiana de Universidades, 1968 , p. 6) y en la mejora de
“(...) la productividad de las universidades prestatarias de este servicio educativo"(...) (Asociación Colombiana de Universidades, 1968, p. 7).

La ES debería masificarse para “(...) democratizar la educación superior, generar igualdad de oportunidades, desarrollar la ciencia y la tecnología y, lo más importante, diversificar la oferta" con “(...) carreras universitarias de corta duración[ [3] tan indispensables en el desarrollo económico de Colombia" (Asociación Colombiana de Universidades, 1968, p.9) y “(...) las auxiliares de las profesiones largas [que] no han alcanzado un desarrollo adecuado" (Asociación Colombiana de Universidades, 1968, p. 129). Por lo tanto, se requerirían al menos dos tipos diferentes de instituciones. Unas, con la finalidad primordial de realizar formación profesional, en tiempo corto (tres años), en una multiplicidad de carreras intermedias auxiliares y cortas (Asociación Colombiana de Universidades, 1968, p. 338); que, en concordancia con la Clasificación Internacional Normalizada de la Educación -aprobada por la Conferencia Internacional de Educación en su 35a reunión (Ginebra, 1975) y la Conferencia General de la Unesco (París, 1978) (Organización de las Naciones Unidas para la Educación, la Ciencia y la Cultura, 2006, p. iii) y conocida actualmente como CINE 1997-, correspondería a las denominadas modalidades de nivel 5.

Es decir, aquellas relativas a un primer ciclo de la educación terciaria no conducen directamente a una calificación avanzada y se dirigen

(...) a segmentos específicos de población que requieren prontamente ingresar al mercado del trabajo. Las calificaciones del nivel 5B suelen exigir menos tiempo que las del $5 \mathrm{~A}$ y se centran en destrezas específicas de una profesión, con miras a ingresar en el mercado de trabajo, aunque el respectivo programa puede abarcar algunas bases teóricas (Organización de las Naciones Unidas para la Educación, la Ciencia y la Cultura, 2006, p. 35).

3 Por "corta duración" léase formación tecnológica. 
A propósito de las recomendaciones de la Universidad de California y del Informe Atcon, el gobierno recurre a nuevas misiones. Esta vez a la de los ingleses Dennis Martin y John Young (1970) y John Talbot (1971-1972), la Misión de la Sociedad Americana para la Enseñanza de la Ingeniería (1971) y la Misión del Gobierno de la Gran Bretaña (1973 y 1974); en esta última recayó la responsabilidad de poner en marcha lo sugerido en el Plan Básico de Educación a propósito de las carreras cortas en el país-que para ese momento ya había asumido su identidad en términos de carreras tecnológicas o educación tecnológica (ET)-, por vía de estructurar un proyecto denominado Bases para un Plan Nacional de Desarrollo de la Educación Tecnológica (1972-1978),que, en líneas con las necesidades que planteaba el desarrollo económico y las problemáticas de la ES, definió los siguientes objetivos (Ministerio de Educación Nacional et al., 1974a, pp. 3-4): 1) fortalecimiento, adecuación y expansión de los institutos; 2) planeamiento y construcción de una red nacional de institutos politécnicos; 3) capacitación del personal docente, técnico y administrativo; 4) creación científica y tecnológica para el país y, al egresado, la vinculación apropiada al mercado del trabajo; 5) buscar la orientación e integración de las instituciones de educación tecnológica existentes; 6) definir la ubicación de la educación tecnológica y 7) un estatuto legal que asegure el desarrollo de la educación tecnológica dentro de los objetivos previstos.

En el marco del plan, la ET fue definida como una “(...) fase culminante de la educación vocacional, tomada esta en su concepción de preparar el ejercicio de una profesión u oficio, más o menos especializado" (Ministerio de Educación Nacional et al., 1974c, pp. 21-22), es decir, en líneas con lo establecido por los organismos internacionales en relación con los programas $5 \mathrm{~B}$, orientados a lo “(...) específico de una profesión (...) para que los participantes adquieran las destrezas prácticas y los conocimientos necesarios para ejercer una profesión particular o un oficio o tipo de profesión u oficio. La aprobación de los correspondientes programas suele facilitar a los participantes la calificación adecuada para el mercado de trabajo" (Organización de las Naciones Unidas para la Educación, la Ciencia y la Cultura, 2006, p. 36).

\section{La institucionalización de la formación tecnológica}

Al amparo de las sugerencias contenidas en el Plan de Desarrollo de la Educación Tecnológica, el gobierno pone en marcha su plan modernizador; para ello creó la dependencia encargada -la División de Educación Tecnológica y Ocupacional (1974), adscrita al recién inaugurado Instituto Colombiano de Fomento a la Educación Superior ${ }^{5}$

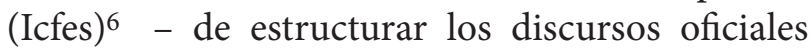
sobre la ET. Así, estableció que la ET, “(...) además de una enseñanza de estudios generales, [involucra] el aprendizaje de ciencias y tecnologías afines y la adquisición de conocimientos prácticos referentes a las ocupaciones especializadas de los sectores industrial, agrícola, comercial y de los sectores análogos así como los servicios sociales y demás aspectos de la economía" (Ministerio de Educación Nacional et al., 1974c, pp. 21-22).

También definió que, en áreas de las tecnologías asociadas a las ingenierías, los programas de ET corresponderían a las ramas de las ingenierías (civil, mecánica, eléctrica, electrónica, química, metalúrgica, etc.), para que los tecnólogos tuvieran “(...) algunos conocimientos del trabajo que tiene que hacer el trabajador manual: están familiarizados con el lenguaje, la maquinaria y las herramientas del obrero y entienden los principios básicos con los cuales trabaja el ingeniero", para traducir las "(...) ideas creativas en nuevos productos, sistemas y procedimientos (...)” (Ministerio de Educación Nacional et al., 1974b, p. 2).

En razón a ello, los propósitos formativos de las carreras deberían encaminarse “(...) principalmente en la aplicación de los métodos científicos

\footnotetext{
5 También en respuesta a las recomendaciones de la Misión Asesora de la Universidad de California.

6 En su calidad de institución que ejerce la suprema vigilancia, reglamentación y control de la educación superior colombiana.
} 
establecidos y no a la búsqueda de nuevos métodos y descubrimientos" (Ministerio de Educación Nacional et al., 1974b, p. 12). En respuesta a esas particularidades, el plan de estudios incluiría “(...) lo más pronto que [fuera] posible, materias técnicas especializadas, habida cuenta del corto tiempo disponible para cubrir la totalidad del programa" (Ministerio de Educación Nacional et al., 1974b, p. 15). Estos programas, de conformidad con lo establecido por los organismos internacionales, tendrían “(...) una duración mínima de 2 años, calculados en tiempo completo, pero con mayor frecuencia de 3 años (...)" (Organización de las Naciones Unidas para la Educación, la Ciencia y la Cultura, 2006, p. 36).

Para continuar con las acciones modernizadoras fue necesario establecer y desplegar los mecanismos de control sobre los programas en general $y$, en especial, sobre los planes de estudio. Así, se despliegan los primeros dispositivos de control del Estado. Un primer decreto (1348 de 1974), de orden general, define que "(...) los establecimientos de Educación Superior son institutos de educación tecnológica cuando sus programas tienen por objeto desarrollar destrezas y habilidades relacionadas con una tecnología específica e impartir los conocimientos generales y científicos en que esta se fundamenta" (Diario Oficial 34141, 1974, artículo 1). Así, indirectamente se define la ET como la encargada del desarrollo de capacidades de orden técnico, pues se invita a pensar que la interacción del tecnólogo, con la tecnología, se limitara al uso de artefactos tecnológicos.

El decreto precisa que “(...) tanto los programas de educación tecnológica como los de corta duración, podrán ser también ofrecidos por las universidades" (Diario Oficial 34141, 1974, artículo 3) y parece pretender diferenciar las carreras tecnológicas -relacionadas con las ramas de la ingeniería- de las de corta duración. Pero al no ofrecer diferencias entre ellas -se presume que en razón a que ambas compartían una característica común, la duración del proceso de formación-, en lo que respecta a los objetivos, contenidos curriculares y, en especial, a las áreas en las cuales pueden ofertarse y los títulos que otorgan, etc., en la práctica se consideraron como equivalentes $y$, por fuerza de la tradición, el concepto de carreras cortas se hizo extensivo al de carreras tecnológicas.

A reforzar ese imaginario también contribuyó el Icfes. Primero, al implementar el programa de actualización y unificación de los planes curriculares de las carreras tecnológicas (1977-1979) ${ }^{7}$ estableciendo -bajo la denominación de carreras tecnológicas- criterios de funcionamiento para las carreras relacionadas con las ingeniería (electrónica, electromecánica) y para otras como preescolar, horticultura y floricultura, enfermería general, agrícola, pecuaria. Y, segundo, al reconocer como instituciones de $\mathrm{ET}^{8}$ algunas para las cuales esa identidad es cuestionable (Colegio de Cultura Femenina, Escuela de Citotecnología). Así, al relacionar la ET con diversas profesiones y áreas del conocimiento, además de las tecnologías en áreas de las ingenierías ${ }^{9}$, se sientan las bases de una confusión histórica, conceptual, curricular, normativa, etc.

El segundo decreto (2267 de 1976)-en líneas con el concepto de ET como formación en destrezas y habilidad- no puede ser otra cosa que una norma de carácter esencialmente ocupacional que sostiene que las carreras tecnológicas constituyen una modalidad terminal de la educación superior (Diario Oficial 34742, 1977, artículo 1) que “(...) tienen los siguientes objetivos educativos: a)

7 Que deberían asumir la totalidad de las instituciones.

8 Instituto Colombiano Jaime Isaza Cadavid, Instituto Tecnológico Pascual Bravo, Instituto Electrónico y de Comunicaciones, Instituto Tecnológico Santandereano, Instituto Tecnológico Universitario del Cesar, Instituto Tecnológico Universitario de Cundinamarca, Instituto Técnico Industrial Antonio J. Camacho, Colegio de Cultura Femenina, Escuela de Citotecnología, Colegio Mayor de Cultura Popular, Instituto Superior de Educación Rural.

9 Civil, Mecánica, Eléctrica, Electrónica, Química, Metalúrgica, etc. 
preparar personal técnico de alto nivel con miras a satisfacer los requerimientos de personal calificado planteados por los procesos de desarrollo socioeconómico del país (...)" (Diario Oficial 34742, 1977, artículo 8).

\section{La formación de auxiliares de ingeniería}

La preocupación por fomentar carreras cortas en ramas de las ingenierías - pero evidentemente con motivaciones distintas a las relacionadas con cumplir las demandas de los organismos internacionales-no fue privativa de las instituciones del Estado. Por el contrario, fue compartida por algunas de las hoy más importantes universidades ${ }^{10}$ públicas del país, que ya habían incursionado en la formación de ingenieros diplomados;"(...) emulando (intentar reproducir) los liderazgos ya reconocidos de Europa y los Estados Unidos en materia de educación superior, las élites regionales del país apostaron por una educación de corte tecnológico, para salir del atraso económico y superar las desigualdades sociales" (Acevedo et al., 2009, p. 274), en programas denominados de auxiliares de ingeniería para formar en el “(...) estudio de las cosas útiles (...) $[y]$ responder a la industrialización del país" (Acevedo et al., 2009, p. 273).

Para establecer los programas de auxiliares de ingeniería (1966),"(...) puente, en la empresa, entre el ingeniero y el obrero (...)" (P83E $\left.\mathrm{E}^{11}-4: 4\right)$, las instituciones por sus propios medios acudieron a la asesoría de “(...) países como Checoeslovaquia, que envió profesores, como Holanda, que envió profesores, e incluso Inglaterra, que envió profesores (...) eran generalmente ingenieros de fábrica o de taller que se llaman en Europa, no eran ingenieros [tradicionales], eran ingenieros de fábrica (...) (P83E4:4) [queríamos] (...) hacer una copia de unos modelos europeos, pero fue una muy mala copia, pues no conseguimos que el tecnólogo sea capaz de hacer desarrollo científico" (P32E-130:130), posiblemente en razón a que para la época el país “(...) apenas [iniciaba el] proceso de industrialización en las ciudades y todavía estaba aislada de los diálogos científicos contemporáneos" (Acevedoetal.,2009, p. 274); además de los propósitos antes declarados -a finales de la década de los setenta e inicios de los ochenta-, las universidades públicas también justificaron la pertinencia de estos programas en razón a la necesidad de formar a la población de “(...) muy bajos recursos, a la que tocaba darle una solución de corta duración, pero que también los proyectara hacia su vida laboral, es decir, que los pudiese cualificar también (...)” (P63E-10:10).

\section{De carrera tecnológica a formación tecnológica}

Los primeros decretos (1348 y 2667) no tuvieron una vigencia prolongada, pues en los albores de los años ochenta el Estado decide dar continuidad a la política modernizadora, que seguían demandando los organismos internacionales, y arremete nuevamente contra la ES organizando un nuevo decreto por el cual se organiza la educación possecundaria. En el marco de este nuevo dispositivo de control las carreras tecnológicas, ahora denominadas carreras de Formación Tecnológica (FT) (Diario Oficial 35465, 1980b, artículo 25), adquieren estatus de una modalidad que debería ofrecer las instrucciones tecnológicas (Diario Oficial 35465,1980 c, artículo 45) y que se “(...) ocuparía del ejercicio de actividades tecnológicas, con énfasis en la práctica y con fundamento en principios científicos" (Diario Oficial 35465, 1980b).

El Decreto Ley 080 del año en curso denominado Reforma de la Educación Post-secundaria, es una concreción más de la concentración de poder en los aparatos del Estado, política cada vez más acentuada en la década de los años setenta y que de una manera evidente se puede demostrar en la estructura educativa actual, la cual ha sido sometida al más audaz proceso de "modernización". Modernización que fortalece las políticas de control del proceso educativo y recupera su orientación ideológica para ponerla en manos de los agentes del gobierno, cuyo poder venía siendo

10 Específicamente, por la Universidad Tecnológica de Pereira.

11 En todos los casos las fuentes denominadas con la letra "P" seguida de un número corresponden a las entrevistas realizadas en el marco del trabajo de campo. 
cuestionado por el análisis objetivo y consciente de las bases magisteriales en sus tres niveles. Debemos confirmarlo citando las palabras del actual ministro de Educación Rodrigo Lloreda: “... hacer que el Ministerio de Educación Nacional asuma la verdadera función de señalar las políticas y orientaciones generales" [se agregó la cursiva] (Calle \& Timana, 1980).

Hay que tener en cuenta que el decreto-ley, en contraposición a las normas que le antecedieron, reconoce la relación de la FT con los principios científicos y que para “(...) afirmar la universidad en sus propósitos científicos y educativos, las instituciones de educación superior estarán abiertas a todas las fuerzas sociales, comunicadas en todos los pueblos del mundo, vinculadas a todos los adelantos de la investigación científica y la tecnología y permeables a todas las manifestaciones del pensamiento científico" (Diario Oficial 35465, 1980b, artículo 7).Pero estos reconocimientos no se dan en razón a una ruptura con el concepto de carreras tecnológicas o ET entendida como una formación en destrezas y habilidades, ni pensando en un desarrollo desde presupuestos endógenos; por el contrario, se dan en razón a la fuerza que adquieren para la época los discursos de resistencia que demandaban de los gobiernos un cambio de dirección del desarrollo de la in-dependencia tecnológica entendida como "(...) la capacidad propia que [permita a los países de la región diseñar y desarrollar] una tecnología más adecuada a sus propios objetivos, más respetuosa de sus propios valores culturales y de sus características ecológicas, más interesada en servir a la satisfacción de las necesidades básicas de su población y más apropiada a su propia constelación de factores y recursos" (Sábato\& MacKenzie, 1982, p. 22).

El decreto-ley olvidó por completo el concepto de carreras cortas, obvió establecer diferencias entre tipos de conocimiento (técnico, tecnológico y científico) y, en especial, en relación con los tipos de instituciones. Así fueron denominadas, indistintamente, instituciones técnicas y tecnológi $\neg$ cas aquellas que antes de la vigencia del decreto venían ofreciendo carreras cortas en administración, educación, arte, legislación, ingeniería, etc. (Gómez, 2002). También reafirmó el carácter terminal y no profesional de la FT y ofreció - de conformidad con la duración de las carreras de nivel 5B, como se indicó líneas arriba- estatuto de ES a la formación técnica profesional12; una “(...) educación predominantemente práctica para el ejercicio de actividades auxiliares o instrumentales concretas" (Diario Oficial 35465, 1980b, artículo 26).

Por supuesto, esta decisión tampoco fue fortuita, dado que el objetivo central del decreto era -por vía de los dispositivos de control-institucionalizar las demandas generadas por los organismos internacionales; “(...) desde el informe Atcon y desde el Plan Básico: lo que se ha llamado la educación ocupacional, técnica y vocacional, fue organi $\neg$ zada de acuerdo con los parámetros que impusieron los orga $\neg$ nismos financieros que contribuyeron a su establecimiento, como el caso del Banco Mundial (...)" (Calle\& Timana, 1980), que sugería la urgencia de contar en corto tiempo con “(...) mano de obra rápida, cierto, eso era como lo fundamental (...)" (P84E-7: 7).

En coherencia con esos fines, las diferencias curriculares entre los programas tecnológicos y los técnicos profesionales no fueron sustanciales (Diario Oficial 35666, 1980), sino que se redujeron a formalidades concernientes al número de unidades de labor académica (ULA) ${ }^{13}$. Para la FT, 2.100 ULA distribuidas en porcentajes por áreas, a saber: campo de fundamentación científica (entre 30\% y $40 \%$ ), campo tecnológico específico ${ }^{14}$ (entre $50 \%$ y $60 \%$ ) y en el campo de formación humanística (por

12 Para la Formación Técnica Profesional, los porcentajes del plan de estudios se establecería de la siguiente manera: Campo de Fundamentación Científica, entre 20-30\%; Campo Técnico, entre 60\%-70\%; Campo de Formación Humanística, por lo menos 10\%. Por su parte, para la Formación Tecnológica en: Campo de Fundamentación científica, entre un 30\% y 40\%; En el campo tecnológico específicos, entre un $50 \%$ y $60 \%$, y En el campo de formación humanística, por lo menos un $15 \%$.

13 La unidad de labor académica (ULA) es la medida del trabajo académico evaluable, realizado por el estudiante a través de las experiencias de aprendizaje previstas en un programa de formación de Educación Superior (Diario Oficial, 1980, artículo 1).

14 De las unidades de labor académica asignadas al campo tecnológico específico, al menos un 50\% deberá corresponder a actividad práctica supervisada. 
lo menos un 15\%),y1.400 para la formación intermedia profesional, distribuidas en campo científico (entre $20 \%$ y $30 \%$ ), campo técnico (entre 60\% y $70 \%$ ) y campo de formación humanística (por lo menos un 10\%). Así, las diferencias entre el tecnólogo y el técnico profesional se redujeron a un par de ULA más de ciencias básicas y un poco menos de práctica y de formación humanística.

Estas diferencias banales en lo que respecta a la estructuración de planes de estudio llevaron a que los planes de estudio de la FT fueran muy similares a los de la formación técnica profesional, se reflejaron también en los perfiles de formación y se magnificaron en el mercado del trabajo, pues los empleadores no contaron con los referentes diferenciadores necesarios para tomar decisiones relacionadas con la vinculación, remuneración y promoción de los egresados de estos niveles (Rojas de Pardo et al., 1984); es decir, para los empleadores, tanto técnicos profesionales como tecnólogos fueron asimilados como mano de obra calificada. En consecuencia, el ámbito laboral (Gómez, 1991) se permeó con un alto grado de sustituibilidad de la fuerza de trabajo y la coexistencia de diferentes acepciones en relación con el tecnólogo, bien sea asumiéndolo como un cuasi ingeniero o no diferenciando sus roles ocupacionales con los del técnico.

En síntesis, las disposiciones del Decreto 080 fueron perversas para la FT y para los propósitos que de ella se esperaban -desde los discursos de resistencia en términos de la generación de unas tecnologías más acordes con las necesidades de la industria nacional-, pues el título de tecnólogo se otorgó en la totalidad de áreas del conocimiento y profesiones, a saber: “(...) médico y cirujano, abogado, ingeniero, arquitecto, filósofo, contador, administrador, biólogo. Los programas en ciencias de la educación [también otorgan el] título de tecnólogo en la respectiva rama profesional" (Diario Oficial 35465, 1980b, artículo 31).

\section{La formación tecnológica en la universidad pública}

Con la expedición del Decreto-ley 080, la universidad pública se ve obligada a acogerse al concepto de FT que promulgan los dispositivos de control -"(...) básicamente una formación de nivel intermedio. Lo tecnológico no existía como saber. Pero además, antes de la Ley 30 , se había planteado que esa formación también era una modalidad y, en cuanto modalidad, las universidades podrían tener formación tecnológica. Retomando esa posibilidad algunas universidades crearon programas de formación tecnológica" (P26E-26:26) - y se aparta de la intención de formar auxiliares de ingeniería y asumir el cambio de denominación del título a tecnólogo.Para ello, el primer paso fue modernizar las estructuras administrativas, reorganizando los programas que estaban “(...) un tanto dispersos, adscritos a una dependencia que se llamaba el politécnico que luego se integra a la universidad, pero sigue teniendo el manejo de politécnico. Pero que a partir de estas disposiciones la universidad los concibe desde otro enfoque y le da el nombre Facultad de Tecnología [1984 ${ }^{15}$ ], para que lo que está adscrito a ella, recae sobre esa formación (...)" (P80E-6:6), es decir, una facultad que asume su identidad en términos de la norma y de la modalidad de ES a ofrecer.

Las disposiciones en materia de FT (ciclos cortos) también fueron acogidas por otras universidades ${ }^{16}$ públicas, que al detenerse a “(...) pensar un poquitico en la razón social de la universidad, uno debe decir, sin lugar a dudas, en una sociedad que es profundamente desigual, es mucho más que pertinente para efectos de inclusión social y oportunidades a los jóvenes. Entonces desde lo institucional uno cree que también son profundamente pertinentes los programas" (P29E-10: 10), y otras 17 que establecen sus objetivos misionales en términos de “(...) llegar a lugares remotos donde realmente por la dificultad que tiene la gente no pueden llegar

15 Esta última denominada anteriormente Instituto Politécnico Universitario. Información recuperada dehttp://www.utp.edu.co/ institucional/40-anos.html

16 Universidad Francisco de Paula Santander.

17 Universidad Industrial de Santander, reconocida como institución de educación superior en 1970, nace con programas de formación tecnológica. 
aquí a la universidad, entonces decide llegar por ejemplo a una vereda a dar esa oportunidad de capacitar a la gente" (P71E-69: 69).Es amparada en la política del "(...) gobierno de Belisario Betancur y en la rectoría de Jaime Luis Gutiérrez [que en la universidad se] inicia la educación a distancia. Entonces en la UIS crea un centro de educación a distancia. Sí, inicialmente fue centro, la condición que le impusieron a educación A Distancia es que los programas que ofrecieran no fueran programas relacionados con las facultades, o sea, tenían que ser en áreas disciplinarias diferentes a las que estaban ofreciendo las facultades (...)” (P64E-1 :1).

La UIS quería incursionar en programas con modalidades diferentes a la presencial y se hicieron algunos estudios anteriores pero nunca se concretó o se abrió la unidad académica responsable, y luego con el impulso y del gobierno de Belisario, con esa política de educación a distancia, en ese momento fue que ya la universidad decidió, bueno, sí, vamos a abrir, y se hicieron los proyectos, tanto de creación en ese momento del centro de educación a distancia como de los primeros programas [se agregó la cursiva]; el primer programa fue tecnología empresarial y luego seguimos con el programa de tecnología agrícola y tecnología pecuaria; una vez que se creó el centro empezó el ofrecimiento, el centro se creó en 1983, tal vez desde la década del setenta habían empezado a hacer estos estudios anteriores que te digo" (P66E-4: 4).

Es decir, la oferta se limitó ${ }^{18}$ a aquellas áreas que no respondían a la tradición de la universidad, pues un grupo de dirigentes de la época “(...) creyó que esto era de segunda categoría y de tercera, entonces como éramos de segunda, ese tipo de formación tecnológica también era de segunda, por eso nos separaron -porque seguimos un poco separados del asunto-, y entonces toda la formación tecnológica como era de segunda clase, pues nos enviaron a una facultad de segunda clase" (P73E-65: 65), a la "(...) Facultad de Estudios a Distancia, que eso fue como por la época del año 85, más o menos, la época de Belisario Betancur, cuando se implementó la educación a distancia, cierto, conozco yo pues de esta facultad, que los programas que nacieron (...) obedecían a una política del gobierno de ese entonces (...)" (P77E-2: 2); uno de esos programas “(...) se llama tecnología de mercadeo agropecuario, tratando de catapultar todas las ventajas del departamento en el sector primario de la economía (...)" (P78E-7: 7).

Así entonces, al concatenar las políticas gubernamentales de la época en materia de educación a distancia con la escasez de algunos servicios, se crearon unos programas de FT “(...) que apuntan a la industria farmacéutica, hacia la producción de medicamentos en el país, entonces en ese momento la Universidad de Antioquia, la Universidad Nacional de Colombia (...) la Universidad de Santander y la Universidad del Atlántico enfocaron sus baterías hacia la producción farmacéutica, o sea que el profesional era un profesional para la industria (...)” (P71E-18-18).Puntualmente se pensó en “(...) contar con un profesional que ejerciera la farmacia comunitaria (...) entonces se (...) pensó que era necesario formar un programa de un nivel tecnológico ¡mas no profesional! para atender esa farmacia pública, porque las funciones que desarrollarían allí no requerían de una formación de un nivel superior (...)” (P71E-21-21).

\section{Conclusiones}

Al instaurarse en los países del Tercer Mundo el discurso del desarrollo, la educación se pone en favor del crecimiento económico y la industrialización. Garantizar la industrialización demandó de la modernización de la Educación Superior mediante la institucionalización de una nueva modalidad para el "desarrollo". La historia de la emergencia de la FT en Colombia se funde con la presencia de los organismos internacionales en la región y con la necesidad que estos develan de fomentar las carreras de ciclo corto, para reducir la "pobreza" y para garantizar que el grueso de la población estuviera preparada para emprender la tarea del "desarrollo" y, particularmente, para la industrialización que se pensó en favor de los “(...) países acreedores

18 Universidad Pedagógica y Tecnológica de Colombia. 
[entiéndase especialmente los Estado Unidos que] sostienen que América Latina debe reconvertir su producción para fabricar productos exportables (...). Sin embargo, la producción que se fomenta no es la nacional, no favorece a la industria local, ni menos aún a los trabajadores del propio país; no se fabrica lo que millones de personas con necesidades insatisfechas requerían como consumidores potenciales ya que no poseen capacidad de compra debido a la falta de ingresos consecuencia del desempleo" (Gallego et al., 2011, p. 446). A la par, algunas universidades públicas del país -también preocupadas por el devenir de la industria nacional- intentaron, en los años sesenta-ochenta, reproducir la formación europea de ingenieros de fábrica o de planta. Propósito al que renuncian en razón al despliegue de los dispositivos de control y al cumplimento de las políticas del gobierno de Belisario Betancur y, por esa vía, a las de los organismos internacionales.

\section{Referencias}

Acevedo, A., Giraldo, N.\& Rodríguez, D. M. (2009). La Universidad Tecnológica de Pereira. En: J. R. Martínez, Memoria de una visión cosmopolita (pp. 253-310). Pereira: Universidad Tecnológica de Pereira, Rudecolombia.

Asociación Colombiana de Universidades. (1968). Plan Básico de la Educación Superior en Colombia. Documentos, vol. I. Bogotá: Imprenta Nacional.

Banco Mundial. (2009). La universidad latinoamericana. Claves para un enfoque conjunto de desarrollo coordinado social, económico y educativo en América Latina. Autor: R. Atcon. Edición digital por Christina Hernández Amaya. Recuperado de http://es.scribd.com/ doc/20891378/La-Universidad-Latinoamericana-clave-para-un-enfoque-The-LatinAmerican-university-a-key-for-an-intergrated-ap

Centro de Estudios Estadounidenses, Departamento de Ciencias Políticas de la Facultad de Ciencias Sociales, Universidad de los Andes, Instituto de Estudios Políticos y Relaciones
Internacionales, Universidad Nacional de Colombia. (2011). 50 años de la Alianza para el Progreso en Colombia: lecciones para el presente. Relatoría del evento. Recuperado de: http://ceecolombia.org/index.php

Diario Oficial 34141. (1974). Decreto 1358 de 1974 (julio 11). Por el cual se dictan normas sobre la Educación Superior. Recuperado dehttp://www.mineducacion.gov.co/1621/articles-104755_archivo_pdf.pdf

Diario Oficial 34712. (1977). Decreto 2667 de 1976. Por el cual se definen y reglamentan las carreras tecnológicas. Recuperado dehttp://www. mineducacion.gov.co/1621/articles-102595_ archivo_pdf.pdf

Diario Oficial 35666. (1980a). Por la cual se reglamentan las Unidades de Labor Académica de que trata el artículo 40 del Decreto extraordinario 80 de 1980 (diciembre 1囚). Recuperado de http://www.mineducacion.gov.co/1621/articles-103283_archivo_pdf.pdf

Diario Oficial 35465. (1980b). Decreto-ley 080 de 1980. Por el cual se organiza el sistema de educación pos-secundaria (febrero 26). Recuperado de: http://www.mineducacion.gov. co/1621/articles-102556_archivo_pdf.pdf

Escobar, A. (1998). La invención del tercer mundo. Construcción y deconstrucción del desarrollo. Bogotá: Norma.

Gallego, M., Gil, F.\& Eggers-Brass, T. (2011). Historia latinoamericana 1700-2005. Sociedades, culturas, procesos políticos y económicos. Argentina: Maipue.

García, A. (2006). La estructura del atraso en América Latina. Hacia una teoría latinoamericana del desarrollo. 4aed. Bogotá: Convenio Andrés Bello.

Gómez, V. M. (1991). La educación técnica y tecnológica en Colombia: análisis crítico y propuesta de modelo alternativo. Bogotá: Departamento de Sociología, Universidad Nacional de Colombia. 
Gómez, V.M. (2002a). Cuatro opciones de política sobre educación técnica y tecnológica. Denominación de instituciones y organización del Sistema de Educación Superior por Ciclos de Formación. Bogotá: Instituto Colombiano para el Fomento de la Educación Superior.. Recuperado de http://acreditacion.udistrital. edu.co/documentos/ICFES/educacion_tecnica_tecnologica.pdf

Jaramillo, J. (1989). Nueva historia de Colombia: la educación durante los gobiernos liberales. 1930-1946. Bogotá: Planeta.

Martínez, A. (2004). La escuela expansiva en América Latina. En: De la escuela expansiva a la escuela competitiva. Dos modos e modernización en América Latina. Barcelona: Anthropos y Convenio Andrés Bello.

Ministerio de Educación Nacional, Instituto Colombiano para el Fomento de la Educación Superior. (1974a). Carreras tecnológicas: una necesidad. Bogotá: Instituto Colombiano para el Fomento de la Educación Superior.

Ministerio de Educación Nacional, Instituto Colombiano para el Fomento de la Educación Superior ICFES. (1974b). Programa de Educación Tecnológica Área Ingenierías. Documento elaborado por Fernando Mejía Valenzuela. Bogotá: Instituto Colombiano para el Fomento de la Educación Superior.

Ministerio de Educación Nacional, Instituto Colombiano para el Fomento de la Educación Superior. (1974c). Plan Nacional de Desarrollo de la Educación Tecnológica. Proyecto de Cooperación Técnica con el Gobierno de la Gran Bretaña. Bogotá: Instituto Colombiano para el Fomento de la Educación Superior.

Molina, M.M. (2008). Introducción al estudio de la universidad latinoamericana. Manizales. Re- cuperado de latinoam.estud.educ

Organización de las Naciones Unidas para la Educación, la Ciencia y la Cultura. (1997). Clasificación Nacional Normalizada de la Educación CINE 1997. Recuperado dehttp://unesdoc. unesco.org/images/0014/001470/147002s.pdf

Orozco, L.E. (1994). Universidad, modernidad y desarrollo humano. Caracas: Centro Regional para la Educación Superior en América Latina y el Caribe.

Ortiz, B. (s.f.). Documento de la línea de investigación Educación Superior y Representaciones Sociales. Énfasis Historia, Pedagogía y Educación Comparada. Doctorado Interinstitucional en Educación (DIE-UD). Recuperado dehttp://die.udistrital.edu.co/sites/default/ files/doctorado_ud/lineas_inv/documentos/ educacion_superior_y_representaciones_sociales_blanca_ortiz.pdf

Pacheco, I. (2002). Evolución legislativa de la educación superior en Colombia. Educación culpable, educación redentora. Digital Observatory for Higher Education in Latin America and the Caribbean. Recuperado de http://unesdoc. unesco.org/images/0013/001399/139967s.pdf

Poveda, G. (1993). Historia social de las ciencias en Colombia. Tomo IV: Ingeniería e historia de las técnicas. Bogotá: Instituto Colombiano para el Desarrollo de la Ciencia y la Tecnología Francisco José de Caldas.

Rojas de Pardo, G., Mora, C. \& Villamizar, M.T. (1984).Diferencias entre las modalidades postsecundarias de formación intermedia profesional, formación tecnológica y formación universitaria en educación pre-escolar (tesis). Maestría en Administración y Supervisión Educativa, Universidad Externado de Colombia, Facultad de Ciencias de la Educación. 